\title{
BMJ Open Patient and stakeholder involvement in resilient healthcare: an interactive research study protocol
}

\author{
Veslemøy Guise (D) , ${ }^{1}$ Karina Aase (D) , ${ }^{1}$ Mary Chambers, ${ }^{2}$ Carolyn Canfield, ${ }^{3}$ \\ Siri Wiig ${ }^{1}$
}

To cite: Guise V, Aase K, Chambers M, et al. Patient and stakeholder involvement in resilient healthcare: an interactive research study protocol. BMJ Open 2021;11:e049116. doi:10.1136/ bmjopen-2021-049116

- Prepublication history for this paper is available online. To view these files, please visit the journal online (http://dx.doi. org/10.1136/bmjopen-2021049116).

Received 18 January 2021 Accepted 25 May 2021

Check for updates

(c) Author(s) (or their employer(s)) 2021. Re-use permitted under CC BY-NC. No commercial re-use. See rights and permissions. Published by BMJ.

${ }^{1}$ SHARE - Centre for Resilience in Healthcare, Faculty of Health Sciences, University of Stavanger, Stavanger, Norway ${ }^{2}$ Centre for Public Engagement, Faculty of Health, Social Care and Education, St George's University of London, London, UK

${ }^{3}$ Department of Family Practice, The University of British Columbia Faculty of Medicine, Vancouver, British Columbia, Canada

Correspondence to

Veslemøy Guise;

veslemoy.guise@uis.no

\section{ABSTRACT}

Introduction Resilience in healthcare (RiH) is understood as the capacity of the healthcare system to adapt to challenges and changes at different system levels, to maintain high-quality care. Adaptive capacity is founded in the knowledge, skills and experiences of the people in the system, including patients, family or next of kin, healthcare providers, managers and regulators. In order to learn from and support useful adaptations, research is needed to better understand adaptive capacity and the nature and context of adaptations. This includes research on the actors involved in creating resilient healthcare, and how and in what circumstances different groups of patients and other key healthcare stakeholders enact adaptations that contribute to resilience across all levels of the healthcare system.

Methods and analysis This 5-year study applies an interactive design in a two-phased approach to explore and conceptualise patient and stakeholder involvement in resilient healthcare. Study phase 1 is exploratory and will use such data collection methods as literature review, document analysis, interviews and focus groups. Study phase 2 will use a participatory design approach to develop, test and evaluate a conceptual model for patient and stakeholder involvement in RiH. The study will involve patients and other key stakeholders as active participants throughout the research process.

Ethics and dissemination The $\mathrm{RiH}$ research programme of which this study is a part is approved by the Norwegian Centre for Research Data (No. 864334). Findings will be disseminated through scientific articles, presentations at national and international conferences, through social media and popular press, and by direct engagement with the public, including patient and stakeholder representatives.

\section{INTRODUCTION}

Resilience in healthcare (RiH) is fundamental to understanding quality in healthcare provision $^{1}$ and is defined by Wiig and colleagues as 'the capacity to adapt to challenges and changes at different system levels, to maintain high-quality care'. ${ }^{2}$ As an emerging field of study within health services research, $\mathrm{RiH}$ views healthcare as a complex adaptive system. Complex adaptive systems are fundamentally characterised

\section{Strengths and limitations of this study}

- This study will contribute to a limited yet growing body of knowledge of patient and stakeholder involvement (PSI) in resilience in healthcare (RiH).

- This study will translate system-wide concepts of resilience into practice by developing and testing a conceptual model for PSI in RiH.

- This study adopts a participatory approach to the development and test of a conceptual model for PSI in RiH, involving stakeholders from a variety of healthcare contexts across all levels of the healthcare system.

- This study features a broad approach to healthcare stakeholders which include patients and family carers, as well as providers, managers and regulators of healthcare services.

- The 5-year project period may restrict opportunities for documenting long-term outcomes of the implementation of the conceptual model for PSI in RiH.

by uncertainty and changing conditions, with multiple individual yet interconnected stakeholders acting in both predictable and unpredictable ways. ${ }^{3}$ In response to inherent uncertainties and highly dynamic conditions, people in complex healthcare systems create and uphold quality and safety by developing and enacting adaptive capacities. This allows them to anticipate and respond to emerging changes, challenges, variations or disruptions to keep the system functioning. ${ }^{24-7}$

The concept of adaptation in complex systems has a variety of interpretations. ${ }^{6}$ Adaptive capacity as a key feature of $\mathrm{RiH}$ may be understood as a dynamic set of internal (ie, cognitive and behavioural) and external (eg, colleagues, networks, regulation) resources used by individuals and teams within an organisational context to adapt everyday functioning according to changing conditions. ${ }^{18}$ As such, adaptive capacity is founded in the knowledge, skills and experiences of the people in the system, and includes systemwide performance adjustments, workarounds, 
trade-offs, sense-making efforts and improvisations. ${ }^{6-9}$ In order to improve healthcare quality, it is imperative to learn from and support useful adaptations. ${ }^{10}{ }^{11}$ Research is therefore needed to better understand adaptive capacity and the nature and context of adaptations-not least the actors involved in creating resilient healthcare. This includes research on how and in what circumstances different groups of patients and other key healthcare stakeholders enact adaptations that contribute to resilience. Research is also needed that explores how patient and stakeholder involvement (PSI) in resilient healthcare can be developed and supported across all levels of the healthcare system. This study seeks to address these gaps in the research knowledge.

\section{PSI in resilient healthcare}

User involvement is a key dimension of healthcare quality that is considered crucial to understand and operationalise $\mathrm{RiH} .{ }^{2}$ The notion of user involvement here is one that emphasises the value and importance of including the knowledge and experiences of a diversity of stakeholders in efforts to understand and improve healthcare services. ${ }^{12}$ A healthcare stakeholder is any person, group or organisation who provides, receives, manages, regulates or pays for healthcare, and can include, for example, patients, family or next of kin, healthcare professionals, managers, regulatory bodies, non-governmental organisations, municipalities and regional authorities. ${ }^{1}$ Traditionally there has been little empirical research focused on contributions to resilient healthcare from patients and stakeholders such as family carers. ${ }^{9}{ }^{13}$ It is increasingly acknowledged, however, that patients and other key stakeholders are important co-creators of $\mathrm{RiH}$, and that without knowledge of how they both facilitate and disrupt RiH, our understanding of everyday clinical work and how best to improve healthcare quality will remain limited. ${ }^{14-16}$

Emerging research indicates that adaptation and responsiveness to variation or changes in complex healthcare systems require the involvement of patients and stakeholders across all levels of the healthcare system. $^{14}{ }^{15}{ }^{17-19}$ Studies have, for example, found that next of kin in cancer care contribute to system resilience and healthcare quality by brokering knowledge between patients and clinicians; bridging gaps between care levels as well as provider organisations; filling gaps on the ward; and otherwise performing tasks that scaffold or support the work of clinicians. ${ }^{20}{ }^{21}$ Similarly, a study of next of kin involvement in regulatory activities found that the information provided by next of kin gives a fuller picture of the cases under investigation and that this type of involvement can lead to more comprehensive and robust regulatory findings. ${ }^{22}{ }^{23}$ Further multistakeholder perspectives are vital to gain more complete knowledge of how to create, sustain and support resilient healthcare. ${ }^{9}{ }^{14}$ More research is also needed on the way PSI in RiH takes place and how to facilitate and support involvement, to improve healthcare quality.

\section{The RiH research programme}

The study on PSI in resilient healthcare described here is part of a comprehensive research programme called RiH. ${ }^{1}$ The objective of the overall $\mathrm{RiH}$ programme is to reform the understanding of quality in healthcare by developing and testing a theoretical and practical $\mathrm{RiH}$ framework. The RiH research programme will be conducted over a 5-year period (2018-2023) and consists of five inter-related work packages, including one focused on the role of collaborative learning in $\mathrm{RiH}^{24}$ and one which concerns an international comparative study of $\mathrm{RiH}$ and teamwork. ${ }^{25}$ This study protocol is limited to the work package concerned with the study of PSI in RiH.

\section{AIM AND RESEARCH OBJECTIVES}

The main aim of this study is to describe how patients and other key stakeholders are involved in creating and sustaining $\mathrm{RiH}$ and how these practices can be supported and enhanced to facilitate high-quality healthcare. The study's primary research question is: 'How can involvement of patients and stakeholders in $\mathrm{RiH}$ be described and improved?' Specifically, the research objectives of this study are as follows:

1. To explore how patients and stakeholders contribute to $\mathrm{RiH}$.

2. To develop a conceptual model for how PSI in RiH can be understood and improved.

3. To test and refine the conceptual model of PSI in RiH across differing empirical contexts using collaborative learning tools and innovations.

To address these objectives, the following research questions will be investigated:

- Which healthcare stakeholders are involved in the creation of RiH in different contexts?

- How and in which contexts do different groups of patients and stakeholders contribute to RiH across different levels of the healthcare system?

- What are the key features of a conceptual model for PSI in RiH, and how can it be tested and validated?

- What are central outcomes of the evaluation of the conceptual model for PSI in RiH?

\section{METHODS AND ANALYSIS \\ Design}

This study on PSI in resilient healthcare has an interactive design. ${ }^{26}$ Interactive research is a participatory approach concerned with developing theoretical knowledge of practical relevance. It is characterised by continuous collaboration and joint learning between the researchers, stakeholders and participants. As such, this study will involve patients, carers, healthcare professionals, regulatory bodies, the public and other major stakeholders (eg, patient organisations, policymakers) as partners throughout the research process, including as co-researchers. The study will be conducted in two phases. Study phase 1 is exploratory and will feature a variety of data collection methods, including literature 


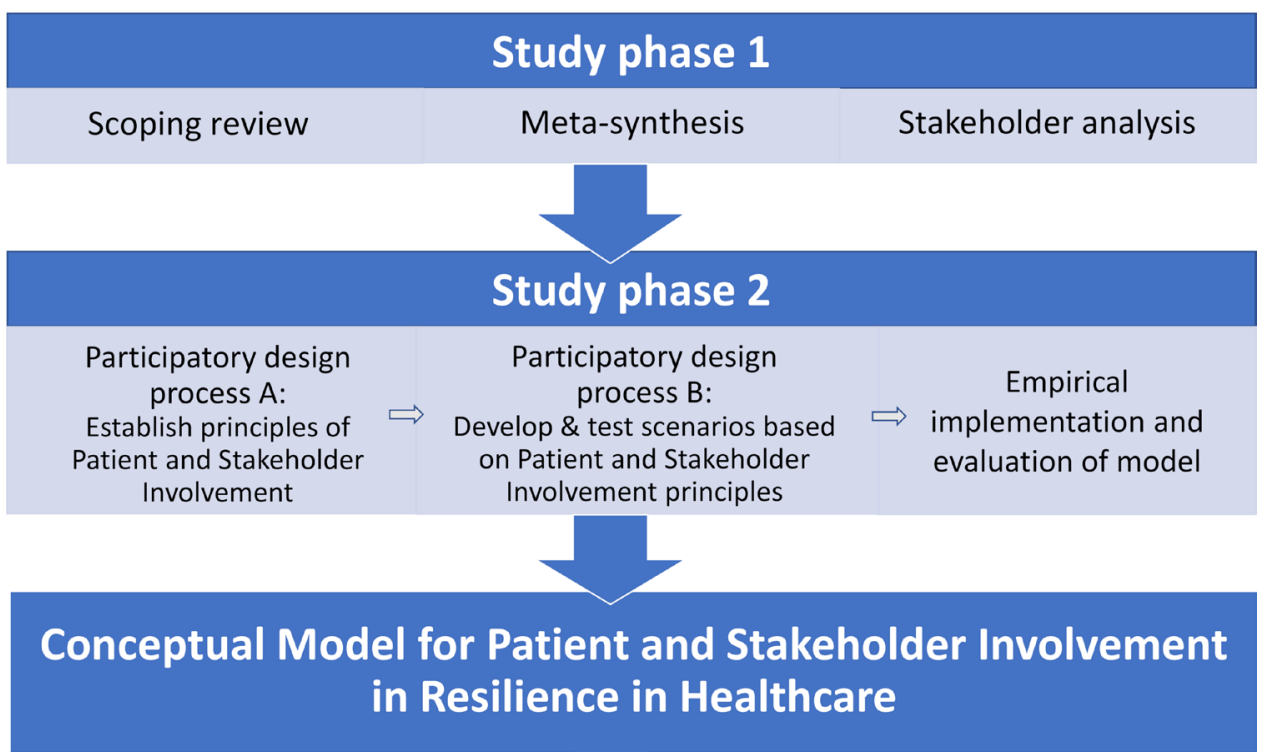

Figure 1 Study phases.

review, meta-synthesis of results from selected empirical studies, interviews and focus groups. Study phase 2 will use a participatory approach to develop, implement and evaluate a conceptual model for PSI in RiH, using focus group and observational data to study impact. See figure 1 for a visual presentation of the study phases.

\section{Study phase 1: exploring patient and stakeholder contributions to RiH}

The purpose of phase 1 is to explore how patients and stakeholders contribute to $\mathrm{RiH}$ and to establish the current knowledge base on PSI in RiH. This will be done by way of a multifaceted approach featuring a scoping review of the literature, an explorative meta-synthesis of findings from a sample of selected empirical projects and a stakeholder analysis.

Scoping reviews are recommended when the purpose of the review is to explore the breadth or depth of the literature on a topic; map and summarise evidence; clarify concepts; identify or address knowledge gaps; and inform future research. ${ }^{27} 28$ The scoping review will focus on patient and family and other caregivers' contributions to $\mathrm{RiH}$ and will seek to answer the question 'How and in which contexts do different groups of patients, families and caregivers contribute to resilience across different levels of the healthcare system?' The scoping review will be conducted according to an established methodological approach. ${ }^{29}$ The chosen search strategy will aim to identify academic output (ie, peer-reviewed journal articles, book chapters, books, conference proceedings), using the following databases: Medline, SCOPUS, ScienceDirect and CINAHL. The search terms to be used are presented in box 1 .

The inclusion criteria are that research items must report on empirical studies with qualitative or mixedmethods designs that describe adaptations, adaptive capacity or organisational resilience in healthcare settings. Furthermore, studies from settings at all levels (micro, meso and macro) of the healthcare system are of interest, and studies must feature patients, families, and/or caregivers as participants. Conversely, research items not from the healthcare setting; without patients, families, or caregivers as participants; and those describing individual or psychological resilience will be excluded. Included items will be restricted to those published in English only. No limits will be set on publication dates. In addition to the electronic searches, we will undertake reference tracking and citation tracking alongside hand searches of key items. The data extraction process will focus on purpose and aim of the studies; study design; participant types and characteristics; type of healthcare service and setting described; the characteristics of identified performance variation or adaptations and the actors involved; and the empirical context of the performance variation or adaptations identified. The results of the scoping review will be reported in line with the Preferred Reporting Items for Systematic Reviews and Meta-Analyses Extension for Scoping Reviews reporting checklist. ${ }^{30}$

For the meta-synthesis, publicly available documents and publications from a broad sample of empirical research projects will be selected according to a set screening protocol, which includes the use of a Quality and Resilience Trigger Tool. The details on this screening protocol and the trigger tool have already been

\section{Box 1 Search terms for the scoping review}

resilien* $O R$ safety* $I$ OR adaptive*capacity $O R$ adapt* $O R$ varia* $O R$ monitor* OR adjust* OR anticipat* OR respon* OR detect* OR learn* AND

patient* OR (carer* or caregiver* or informal carer* or family member $^{*}$ or relative* or next-of-kin)

AND

healthcare $O R$ health care

*=wildcard filter. 
published as part of the overall $\mathrm{RiH}$ research programme protocol. ${ }^{1}$ Specifically, the sample will be drawn from a pool of approximately 50 research projects, including post-doctoral projects and $\mathrm{PhD}$ projects, which involve researchers from the SHARE-Centre for Resilience in Healthcare and were conducted in Norway between 2010 and 2021 (some are ongoing). The screening process will establish if and how the projects relate to resilience and which healthcare quality components they cover. After screening, a total sample of around 20 empirical projects will be selected to reflect a comprehensive range of healthcare settings, stakeholders, quality dimensions (ie, patient safety, continuity of care, patient-centeredness, clinical effectiveness), and adaptive capacities at individual, team/unit, organisational, and larger system level.

The stakeholder analysis $^{31}$ will be undertaken in a selected sample of empirical healthcare studies with the aim of mapping relevant actors in the sample by identifying who they are and exploring their roles, interests, needs and inter-relations, where in the system they are involved, how and in which contexts they are involved in $\mathrm{RiH}$, and what type of adaptive capacities they are involved in. We will also explore the influences and resources they may exert on performance variability and how adaptations are enacted to facilitate $\mathrm{RiH}$ in certain contexts. The knowledge generated in the stakeholder analysis will be used to identify stakeholders suitable for involvement in the design and development of the conceptual model, and also to better understand the context in which the model will be tested and implemented. ${ }^{32}$ Aside from helping to inform appropriate planning and development processes, conducting a stakeholder analysis is useful as part of building a collaborative approach to the development and delivery of healthcare services and healthcare innovations. ${ }^{33}$

\section{Participants}

This phase of the study will draw participants from a total sample of 30-40 researchers involved in completed, ongoing, or newly initiated empirical research projects with a theoretical and/or empirical connection to PSI and/or RiH. As noted above, the sampling process will be project focused and entail a screening process based on publicly available information about relevant projects. This will establish how the projects relate to resilience and which healthcare quality components they encompass. ${ }^{1}$ Once the sample of projects has been established, the researchers involved in the included projects will be approached and recruited to participate in the study using one or more of the data collection methods described below.

\section{Data collection}

The data collection process during this part of the study will, as noted above, feature meta-synthesis of findings from existing empirical projects, using publicly available material such as peer-reviewed articles, book chapters, $\mathrm{PhD}$ theses and project reports. The meta-synthesis will focus on findings related to the links between PSI and RiH. Data collection in the stakeholder analysis will entail individual interviews with 10-15 researchers concerning findings, activities and experiences related to PSI and $\mathrm{RiH}$, in either the planning and/or execution of their research projects, as well as focus group interviews with researchers (approximately three to four interviews with five to seven participants) to brainstorm and categorise stakeholders and their roles, interests, inter-relations and influence. ${ }^{34}$ A range of topics related to involvement in $\mathrm{RiH}$ will be explored during these interviews, including who, how and in which situations patients and stakeholders are involved in and contribute to resilient healthcare; types of involvement; drivers and barriers to involvement; and contextual factors surrounding patients' and other stakeholders' contributions to RiH. Finally, data collection during this study phase will include observations of different types of activities in ongoing projects, such as workshops, stakeholder meetings, co-creation activities, etc to better understand links between PSI and various aspects of RiH through practical examples.

\section{Data analysis}

The data analysis in the meta-synthesis will combine both a thematic inductive approach and a deductive approach based on four key questions deemed central to understand and operationalise $\mathrm{RiH}$ in both research and practice. ${ }^{2}$ These four questions are resilience for what, to what, of what and through what. This combining of inductive and deductive approaches is to ensure identification of key elements of both involvement and adaptive capacity central to RiH. For the meta-synthesis of empirical research projects, data will be analysed according to the following steps, to ensure a sound analytical process across different study designs and empirical settings:

1. Development of short narratives. A narrative of three to four pages will be written for each study included in the meta-analysis. The narrative content will focus on answering the four resilience questions: resilience for what, to what, of what and through what? The narratives will also identify study settings, levels, context, stakeholders, contributions and adaptations.

2. Analysis of the resilience narratives according to an inductive thematic synthesising approach. All narratives that are generated in step 1 will be subject to an inductive thematic synthesis to identify the key stakeholders involved in creating and supporting adaptive capacity at different system levels, and to establish knowledge of the ways different groups of patients and stakeholders contribute to adaptations across various healthcare settings, including important contextual conditions for supporting and enhancing their involvement in resilient healthcare.

Qualitative data from interviews, focus groups and observations will be analysed using a combined thematic inductive approach and a deductive approach according to the same four key questions mentioned above. The totality of the data material collected in phase 1, including 
the outcomes of the scoping review, the meta-synthesis and the qualitative data in the stakeholder analysis, will be combined to provide a foundation for developing the conceptual model for PSI in RiH. A procedure of joint display of data ${ }^{35}$ will guide the process of combining diverse data sources during this analysis.

\section{Study phase 2: develop, test and evaluate a conceptual model for PSI in RiH}

The purpose of phase 2 is to develop a conceptual model for how PSI in RiH can be understood and improved, and to test and evaluate the model using collaborative learning tools and innovations. Findings from phase 1 will feed into phase 2, where the study will apply experiencebased co-design ${ }^{36}$ principles in a two-part participatory process (A and B) to develop the conceptual model based on a synthesising of the prior findings on how patients and stakeholders contribute to adaptive capacity, and how the drivers for their involvement can be understood. Following their identification as part of the stakeholder analysis in study phase 1, key actors will be involved in the process to develop the model. The conceptual model will then be tested and validated through a set of learning components (eg, structured meeting arenas, simulation scenarios, interactive digital guides, webinars, etc) in close collaboration with the work package focused on developing knowledge of the role of collaborative learning in $\mathrm{RiH} .{ }^{24}$ Patients and other stakeholders will be involved as co-researchers in the entire process.

The development of the conceptual model for PSI in $\mathrm{RiH}$ will entail a two-part participatory design process, ${ }^{36}$ which will involve a broad group of stakeholder representatives (eg, patients, next of kin, healthcare professionals, managers, policymakers, regulators) and where the main aim is to operationalise the findings from study phase 1 . In the first part of this process (A), principles for PSI in $\mathrm{RiH}$ will be established through a consensus process. This will include principles for how to support and strengthen stakeholder involvement to facilitate adaptive capacity at the individual, team and intraorganisational level. The second part of the participatory design process (B) will then aim to develop prototype simulation scenarios using the PSI principles established during process A, at each level of the healthcare system (ie, micro, meso, macro). The prototype scenarios will be pilot tested in simulation centres at the University of Stavanger and the Norwegian University of Science and Technology in Gjøvik, both of which are partners in the $\mathrm{RiH}$ research programme.

Based on the results from the pilot testing, the scenarios will be further refined and will constitute the basis for further implementation and evaluation of the conceptual model in three empirical settings representing different clinical, managerial and regulatory areas across all levels of the Norwegian healthcare system. The first setting will have a focus on patient and stakeholder contributions to individual and team-based adaptive capacities at the micro-level. The second setting will focus on patient and stakeholder contributions to organisational adaptive capacities at the meso-level. The third setting will focus on patient and stakeholder contributions to intraorganisational adaptive capacities at the macro-level. A RiH laboratory will be established to assist these implementation activities. The RiH laboratory will combine digital learning formats, such as internet-based resources, virtual simulation scenarios, podcasts, etc with testing at the noted simulation centres as well as in situ simulation in clinical settings. The implementation and evaluation of the conceptual model for PSI in RiH will take place over a 12-month period. Both positive and negative impacts of the conceptual model will be appraised using a participant observation approach as part of a process evaluation. $^{3738}$

\section{Participants}

The consensus process in participatory design process A will involve 15-20 stakeholder representatives from various settings across different levels of the healthcare system. These participants will be selected in accordance with the findings from the stakeholder analysis in study phase 1, but will likely include patients, family or next of kin, healthcare professionals, managers, policymakers and regulators. For participatory design process B, approximately 15-20 participants will be recruited, including clinical experts, education experts and representatives from different levels of the healthcare system, as well as patients and family or next of kin representatives from different clinical settings like hospitals, home healthcare services, nursing homes, general practice and prehospital services.

Participants in the subsequent implementation and evaluation process will be recruited from the three different empirical settings in Norway where the conceptual model for PSI in RiH is to be tested. The participants in this part of the study will thus include end users from these three, yet-to-be determined healthcare settings, representing all three levels of the healthcare system (eg, patients and healthcare professionals at the micro-level, managers at the meso-level and regulators at the macrolevel). Around 25-30 participants will be involved in testing and evaluating the conceptual model at each of the three separate empirical settings, meaning that a total of approximately 70-90 participants will be part of the testing of the conceptual model.

\section{Data collection}

For the first participatory design process (A), the data collection will be focused around the consensus process to establish principles for PSI in RiH. During this consensus process, the research team will conduct consensus meetings, workshops and focus group interviews with key stakeholders. Around four to seven meetings and workshops and four to seven focus group interviews, each with four to eight participants, will be undertaken. Themes for the workshops and meetings will be set based on the findings from phase 1, though with a likely focus on, for example, who the patients and stakeholders are, how they 
contribute to adaptive capacity and in which contexts, and how to strengthen their involvement in useful adaptations.

As part of the second participatory design process (B), which as noted is concerned with developing prototype simulation scenarios based on the conceptual model's principles of PSI, scenarios will be tested and refined in the involved simulation centres using an iterative process. During this process, data will be collected through observations and focus group interviews with different participants as described above. We estimate approximately 20 hours of observations and four to six focus group interviews with five to seven participants in each will be conducted to refine the prototype scenarios.

Data collection during the subsequent implementation and evaluation of the principles of the PSI conceptual model in empirical practice will include observation, focus group interviews and individual interviews. Data will be collected at three separate stages of the implementation process: prior to implementation, halfway through and at the end of the implementation period. Around 10-15 interviews and 2-3 focus groups with 5-7 participants each are envisaged before, during and after implementation for each of the three empirical settings involved. In addition, we will conduct observations of the implementation process itself to understand the impact of PSI in the collaborative learning activities. The main purpose of the interviews and observation is to understand how the model for PSI in RiH functions in different empirical settings.

\section{Data analysis}

The process evaluation will integrate findings from the interviews, focus group interviews and observation. The procedure of joint display of data ${ }^{30}$ will be applied to identify how patients and stakeholders contribute to adaptive capacity, and how to support their involvement in RiH to enable adaptive capacity at the individual, team and intraorganisational level.

\section{Patient and public involvement}

Patient and public involvement representatives were involved in the development of the original funding proposal for the $\mathrm{RiH}$ research programme. ${ }^{1}$ The $\mathrm{RiH}$ research programme has a patient and citizen representative as co-chair of its international Expert Advisory Board, who has been involved in the overall project development and has contributed to key aspects of its design. This patient and citizen representative is also a coauthor (author CC) on this paper and has contributed to the design of this dedicated PSI work package. This work package aims to systematically involve patients and other key stakeholders from the Norwegian healthcare system in the overall research programme in multiple ways, for example, as representatives on PSI panels, which will be consulted throughout the conduct of this study, and as study participants and co-researchers in a collaborative, interactive research process. Patients and other stakeholders will also be involved in publications, as well as public engagement activities and in translating research into practice.

\section{Limitations}

There are some potential limitations to this study. First, it must be noted that the outcomes of the empirical implementation and evaluation of the conceptual model of PSI in resilient healthcare may have limited transferability beyond the particular empirical healthcare settings in which the principles of the conceptual model are to be tested. Second, the lack of involvement of patient stakeholders from the Norwegian context at the planning and design stage of the studies featured in the overarching $\mathrm{RiH}$ research programme can be considered a limitation. However, the decision to apply an international perspective at the design stage was made to ensure that the PSI work package commits to extensive engagement of patients and stakeholders that meets international expectations for exemplary participation in all facets of the research. As noted above, this is a work package dedicated to PSI, which will see Norwegian stakeholders including patient and family representatives be involved throughout the remainder of the research process.

\section{ETHICS AND DISSEMINATION \\ Ethics}

The overall $\mathrm{RiH}$ research programme, of which this study is a part, is approved by the Norwegian Centre for Research Data (NSD) (reference number: 864334). As per NSD requirements, a formal 'notification of changes' will be submitted on completion of study phase 1, when further details on the data collection (populations, settings, sampling, etc) in study phase 2 have been established. NSD will then assess the reported changes to establish if they have any consequences for the ethics approval already granted, for example, in relation to the handling and processing of data to be collected in phase 2 .

\section{Dissemination}

This study on PSI in resilient healthcare is part of the $\mathrm{RiH}$ research programme, which has a publication and dissemination strategy focused on the sharing of scientific knowledge, information and results, and on public relations including relevant patient and stakeholder representatives. ${ }^{1}$ Findings from this patient and stakeholder study will first and foremost be disseminated in articles published in peer-reviewed journals, as well as at national and international conferences. In addition, study outcomes will be disseminated to audiences outside the academic community through public presentations and popular science contributions in, for example, social media or in the press. The overall research programme will also organise annual RiH research seminars for academic partners, empirical partners and stakeholders. Annual RiH patient/stakeholder seminars will contribute to the creation of collaborative learning arenas involving 
relevant stakeholders in the Norwegian healthcare system and $\mathrm{RiH}$ researchers, to enable translation of research evidence into interventions for stakeholder use.

Contributors VG and KA developed the initial idea for the study protocol. VG is the principal investigator of the PSI work package and drafted the original manuscript and contributed to manuscript revisions. KA and SW contributed substantial intellectual content to the study design, helped draft parts of the original manuscript and contributed to the revision of the manuscript. MC and CC contributed to the study design and to significant manuscript revisions. All authors have approved the final version of the manuscript.

Funding This work was supported by The Norwegian Research Council (NFR) grant number 275367. The University of Stavanger, the Norwegian University of Science and Technology in Gjøvik, and the Norwegian Air Ambulance Foundation support the programme with in-kind funding.

Competing interests None declared.

Patient and public involvement Patients and/or the public were involved in the design, or conduct, or reporting, or dissemination plans of this research. Refer to the Methods section for further details.

Patient consent for publication Not required.

Provenance and peer review Not commissioned; externally peer reviewed.

Open access This is an open access article distributed in accordance with the Creative Commons Attribution Non Commercial (CC BY-NC 4.0) license, which permits others to distribute, remix, adapt, build upon this work non-commercially, and license their derivative works on different terms, provided the original work is properly cited, appropriate credit is given, any changes made indicated, and the use is non-commercial. See: http://creativecommons.org/licenses/by-nc/4.0/.

\section{ORCID iDs}

Veslemøy Guise http://orcid.org/0000-0002-9124-1664

Karina Aase http://orcid.org/0000-0002-5363-5152

\section{REFERENCES}

1 Aase K, Guise V, Billett $\mathrm{S}$, et al. Resilience in healthcare $(\mathrm{RiH})$ : a longitudinal research programme protocol. BMJ Open 2020;10:e038779.

2 Wiig S, Aase K, Billett S, et al. Defining the boundaries and operational concepts of resilience in the resilience in healthcare research program. BMC Health Serv Res 2020;20:330.

3 Plsek PE, Greenhalgh T. The challenge of complexity in health care. BMJ 2001;323:625-8.

4 Hollnagel E, Wears RL, Braithwaite J. From Safety-I to Safety-II: a white paper. The resilient health care net. USA; Australia: University of Southern Denmark, University of Florida; Macquarie University, 2015.

5 Bergstöm J, Dekker S. The 2010s and onward: Resilience engineering. In: Dekker S, ed. Foundations of safety science: a century of understanding accidents and disasters. Routledge, 2019: 391-429.

6 Foster CJ, Plant KL, Stanton NA. Adaptation as a source of safety in complex socio-technical systems: a literature review and model development. Saf Sci 2019;118:617-31.

7 Anderson JE, Ross AJ, Macrae C, et al. Defining adaptive capacity in healthcare: a new framework for researching resilient performance. Appl Ergon 2020;87:103111.

8 Berg SH, Aase K. Resilient characteristics as described in empirical studies on health care. In: Wiig S, Fahlbruch B, eds. Exploring Resilience - A Scientific Journey from Practice to Theory. Cham: Springer Open, 2019: 79-89.

9 Berg SH, Akerjordet K, Ekstedt M, et al. Methodological strategies in resilient health care studies: an integrative review. Saf Sci 2018;110:300-12.

10 Anderson JE, Ross AJ, Back J, et al. Implementing resilience engineering for healthcare quality improvement using the CARE model: a feasibility study protocol. Pilot Feasibility Stud 2016;2:61.

11 Anderson JE, Ross AJ, Back J. Resilience engineering as a quality improvement method. In: Wiig S, Fahlbruch B, eds. Exploring Resilience - A Scientific Journey from Practice to Theory. Cham: Springer Open, 2019: 25-31.

12 Tritter JQ, McCallum A. The snakes and ladders of user involvement: moving beyond Arnstein. Health Policy 2006;76:156-68.
13 Ellis LA, Churruca K, Clay-Williams R, et al. Patterns of resilience: a scoping review and bibliometric analysis of resilient health care. Saf Sci 2019;118:241-57.

14 O'Hara JK, Canfield C, Aase K. Patient and family perspectives in resilient healthcare studies: a question of morality or logic? Saf Sci 2019;120:99-106.

15 O'Hara JK, Aase K, Waring J. Scaffolding our systems? Patients and families 'reaching in' as a source of healthcare resilience. BMJ Qual Saf 2019;28:3-6.

16 Canfield C. Recovery to resilience: a patient perspective. In: Braithwaite J, Wears RL, Hollnagel E, eds. Resilient health care: reconciling work-as-imagined and work-as-done. Boca Raton, FL: CRC Press, 2017: volume 3. 27-36.

17 Laugaland K, Aase K. The demands imposed by a health care reform on clinical work in transitional care of the elderly: a multi-faceted Janus. In: Wears RL, Hollnagel E, Braithwaite J, eds. Resilient health care: the resilience of everyday clinical work. Farnham: Ashgate, 2015: volume 2. 39-58.

18 Schubert CCet al. Patients as a source of resilience. In: Wears RL, Hollnagel E, Braithwaite J, eds. Resilient health care: the resilience of everyday clinical work. Farnham: Ashgate, 2015: Vol 2. 207-25.

19 Fylan B, Armitage G, Naylor D, et al. A qualitative study of patient involvement in medicines management after hospital discharge: an under-recognised source of systems resilience. BMJ Qual Saf 2018;27:539-46.

20 Bergerød IJ, Gilje B, Braut GS, et al. Next-of-kin involvement in improving hospital cancer care quality and safety - a qualitative cross-case study as basis for theory development. BMC Health Serv Res 2018;18:324.

21 Bergerød IJ, Braut GS, Wiig S. Resilience from a stakeholder perspective: the role of next of kin in cancer care. J Patient Saf 2020;16:e205-10.

22 Wiig S, Haraldseid-Driftland C, Zachrisen RT, et al. Next of Kin Involvement in Regulatory Investigations of Adverse Events That Caused Patient Death: A Process Evaluation (Part I - The Next of Kin's Perspective). J Patient Saf 2019.

23 Wiig S, Schibevaag L, Zachrisen RT, et al. Next-of-kin involvement in regulatory investigations of adverse events that caused patient death (Part II: the inspectors' perspective). J of Patient Saf 2019.

24 Haraldseid-Driftland C, Aase K, Wiig S, et al. Developing a Collaborative Learning Framework for Resilience in Healthcare - A Study Protocol. BMJ Open.

25 Anderson JE, Aase K, Bal R, et al. Multilevel influences on resilient healthcare in six countries: an international comparative study protocol. BMJ Open 2020;10:e039158.

26 Svennson L, Ellström PE, Brulin G. Introduction - on interactive research. Int J Action Res 2007;3:233-49.

27 Peters MDJ, Godfrey CM, Khalil H, et al. Guidance for conducting systematic scoping reviews. Int J Evid Based Healthc 2015;13:141-6.

28 Tricco AC, Lillie E, Zarin W, et al. A scoping review on the conduct and reporting of scoping reviews. BMC Med Res Methodol 2016;16:15

29 Peters MDJ, Marnie C, Tricco AC, et al. Updated methodological guidance for the conduct of scoping reviews. JBI Evid Synth 2020;18:2119-26.

30 Tricco AC, Lillie E, Zarin W, et al. PRISMA extension for scoping reviews (PRISMA-ScR): checklist and explanation. Ann Intern Med 2018;169:467-73.

31 Varvasovszky Z, Brugha R. A stakeholder analysis. Health Policy Plan 2000;15:338-45.

32 Franco-Trigo L, Fernandez-Llimos F, Martínez-Martínez F, et al. Stakeholder analysis in health innovation planning processes: a systematic scoping review. Health Policy 2020;124:1083-99.

33 Franco-Trigo L, Marqués-Sánchez P, Tudball J, et al. Collaborative health service planning: a stakeholder analysis with social network analysis to develop a community pharmacy service. Res Social Adm Pharm 2020;16:216-29.

34 Reed MS, Graves A, Dandy N, et al. Who's in and why? A typology of stakeholder analysis methods for natural resource management. $J$ Environ Manage 2009;90:1933-49.

35 Creswell JW. A Concise introduction to mixed methods research. Los Angeles, CA: SAGE Publications, 2014.

36 Bate P, Robert G. Bringing user experience to healthcare improvement: the concepts, methods and practices of experiencebased design. Oxford: Radcliffe Publishing, 2007.

37 DeWalt KM, DeWalt BR. Participant observation: a guide for fieldworkers. Walnut Creek, CA: Alta Mira Press, 2002.

38 Moore GF, Audrey S, Barker M, et al. Process evaluation of complex interventions: medical Research Council guidance. BMJ 2015;350:h1258. 